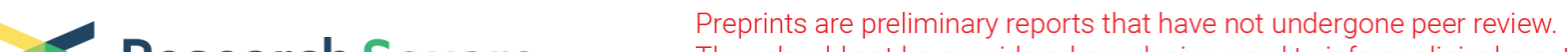 Research Square They should not be considered conclusive, used to inform clinical practice, or referenced by the media as validated information.
}

\section{Neuroanatomical Alterations in Patients With Tinnitus Before and After Sound Therapy: A Combined VBM and SCN Study}

\section{Xuan Wei}

Capital Medical University Affiliated Beijing Friendship Hospital Department of Radiology Han Lv ( $D$ chrislvhan@126.com )

Capital Medical University Affiliated Beijing Friendship Hospital Department of Radiology https://orcid.org/0000-0001-9559-4777

\section{Qian Chen}

Capital Medical University Affiliated Beijing Friendship Hospital

\section{Zhaodi Wang}

Capital Medical University Affiliated Beijing Friendship Hospital

Chunli Liu

Capital Medical University Affiliated Beijing Friendship Hospital

\section{Pengfei Zhao}

Capital Medical University Affiliated Beijing Friendship Hospital

\section{Shusheng Gong}

Capital Medical University Affiliated Beijing Friendship Hospital

\section{Zhenghan Yang}

Capital Medical University Affiliated Beijing Friendship Hospital

\section{Zhenchang Wang}

Capital Medical University Affiliated Beijing Friendship Hospital Department of Radiology

\section{Research}

Keywords: Tinnitus, sound therapy, VBM, structural covariance network, fMRI

Posted Date: September 15th, 2020

DOl: https://doi.org/10.21203/rs.3.rs-70719/v1

License: (c) (1) This work is licensed under a Creative Commons Attribution 4.0 International License. Read Full License 


\section{Abstract}

Background: Many neuroanatomical alterations have been detected in patients with tinnitus in previous studies. However, little is known about morphological and structural covariance network (SCN) changes before and after long-term sound therapy. This study aimed to explore alterations in brain anatomical and SCN changes in patients with idiopathic tinnitus using voxel-based morphometry (VBM) analysis 24 weeks before and after sound therapy.

Methods: Thirty-three tinnitus patients underwent magnetic resonance imaging scans at baseline and after 24 weeks of sound therapy. Twenty-six age- and sex-matched healthy control (HC) individuals also underwent two scans over a 24-week interval; 3.0T MRI and high-resolution 3D structural images were acquired with a 3D-BRAVO pulse sequence. Structural image data preprocessing was performed using the VBM8 toolbox. The Tinnitus Handicap Inventory (THI) score was acquired in the tinnitus group to assess the severity of tinnitus and tinnitus-related distress. Two-way mixed model analysis of variance (ANOVA) and post hoc analyses were performed to determine differences between the two groups (patients and HCs) and between the two scans (at baseline and at the 24th week). Two-sample $t$ tests, paired-samples $t$ tests, and Pearson's correlation analysis were used in the post hoc analysis.

Results: Interaction effects between the two groups and the two scans demonstrated significantly different gray matter (GM) volume in the right parahippocampus gyrus, right caudate, left superior temporal gyrus, left cuneus gyrus and right calcarine gyrus; we found significantly decreased GM volume in the above five brain regions among the tinnitus patients before sound therapy (baseline) compared to that in the $\mathrm{HC}$ group. The 24-week sound therapy group demonstrated significantly greater brain volume compared with the baseline group among these brain regions. We did not find significant differences in brain regions between the 24-week sound therapy and HC groups. The SCN results showed that the left superior temporal gyrus and left rolandic operculum were significantly different in nodal efficiency, nodal degree centrality and nodal betweenness centrality after FDR correction. Decreased THI scores and GM volume changes between the left thalamus and right thalamus were not correlated.

Conclusions: This study characterized the effect of sound therapy on brain GM volume, especially in the left superior temporal lobe. Notably, sound therapy had a normalizing effect on tinnitus patients.

\section{Background}

Tinnitus is a very common otological disorder. Approximately $10 \%$ to $25 \%$ of the population is severely affected $(1,2)$. Tinnitus reduces quality of life for millions worldwide (3). Chronic tinnitus can cause a series of problems, such as sleep disturbances (4), cognitive problems, depression (5), and work disorders (6). Previous studies have shown that tinnitus can cause significant changes in brain function and structure, which are closely related to the clinical manifestations of patients $(7,8)$. In addition, studies have shown that the brain structure and function of tinnitus patients have undergone significant 
remodeling $(9,10)$. Therefore, it is very important to fully understand the abnormal brain nerve activity related to tinnitus.

According to our previous research, tinnitus has been proven to be a symptom characterized by abnormal resting-state functional magnetic resonance imaging (rs-fMRI) (11-14). The brain regions involved include sound detection regions, such as the insula and hippocampus $(15,16)$, and auditory and nonauditory brain regions (17), such as the parahippocampal gyrus (18), posterior cingulate cortex (19), and anterior cingulate cortex (20). In recent years, the study of brain microstructure has also received increasing attention. Our previous research also proved that tinnitus can result in significant alterations in brain white matter (WM) microstructure (10).

Indeed, microstructural changes in the brain have also been reported in some tinnitus studies $(21,22)$. Different studies have used different methods of measuring microstructure. Voxel-based morphometry (VBM) is a neuroimaging technique that investigates focal differences in brain anatomy (23); it can quantitatively detect the volume of brain tissue at the voxel level, reflecting the differences in the components and characteristics of brain tissue in local brain regions of different groups or individuals (24). Currently, VBM has been used increasingly widely to describe microstructural changes in the brain in tinnitus patients $(25,26)$. A meta-analysis revealed structural alterations in the brain of tinnitus patients in the superior temporal gyrus, middle temporal gyrus (MTG), angular gyrus, caudate nucleus, superior frontal gyrus, and supplementary motor area (27). Our previous study also showed that compared with normal controls, patients with unilateral pulsatile tinnitus have a significantly increased gray matter (GM) volume in the bilateral superior temporal gyri (28). At the same time, structural brain networks (SCNs) were widely used in behavioral research and other diseases $(29,30)$. However, this method was not used in the evaluation of the treatment efficacy of tinnitus. In this study, SCNs were obtained using graph theoretical analysis. After obtaining the volume of GM in the first step, we continued to analyze its SCNs, to further evaluate the results.

A deep understanding of the functional and anatomical changes of the brain is a key factor for the effective treatment of tinnitus. Many treatment modalities have been applied to tinnitus patients, such as those treated with repetitive transcranial magnetic stimulation (rTMS) (31), drug therapy (32), tinnitus counseling and cognitive behavioral therapy (CBT) (33), hearing aids (34), cochlear implants (35), and tinnitus retraining therapy (36). Krick applied music therapy to observe patients' GM volume. After the Heidelberg model of music therapy intervention, the GM of the precuneus, medial superior frontal areas, and auditory cortex increased in acute tinnitus patients accompanied by significantly decreased tinnitusrelated distress (37). In recent years, narrow band noise sound therapy has been one of the commonly used methods for the treatment of tinnitus (38). Our previous studies have demonstrated functional changes in the brain with this sound therapy $(8,39)$. However, we only found a few related reports on the morphological changes before and after sound therapy $(37,40)$.

In this study, tinnitus patients who underwent 24 weeks of narrow band noise sound therapy were enrolled. VBM and SCNs were applied to analyze the anatomical changes in the brain in patients before 
sound therapy and after sound therapy as well as to acquire data from healthy controls (HCs) at baseline and at 24 weeks to explore the morphological features and network alterations. We hypothesize that the brain regions associated with tinnitus, involving auditory, attentional, subcortical systems and other regions, especially the superior temporal gyrus, may show volume and network alterations after sound therapy. This study will help provide deeper insight into the changes in the brain after long-term treatment for tinnitus from a neuroanatomical perspective.

\section{Methods}

\section{Standard protocol approval, registration and patient consent}

This research involved human participants. All authors have declared that this research was approved by the Institutional Review Board (IRB). This study was approved by the ethics committees of our research institution (Beijing Friendship Hospital, Capital Medical University, 2016-P2-012). Written informed consent was obtained from all study subjects.

\section{Subjects}

All patients and healthy volunteers were recruited in our institution. In this study, thirty-three patients with idiopathic tinnitus were enrolled. The tinnitus sound was described as a persistent, high-pitched sound in both ears. The inclusion criteria were as follows: (1) 18 to 65 years old; (2) right handedness; (3) tinnitus duration ranging from 6 to 48 months; (4) no significant hearing loss (hearing thresholds $\geq 25 \mathrm{~dB} H \mathrm{HL}$ at frequencies of $0.250,0.500,1,2,3,4,6$, and $8 \mathrm{kHz}$ determined by pure tone audiometry (PTA) examination; and (5) willingness to receive sound therapy for 24 weeks and then return to be re-examined after treatment. The exclusion criteria included (1) other kinds of tinnitus (such as pulsatile tinnitus), Meniere's disease, sudden deafness, or otosclerosis; (2) neurological signs and/or a history of neurological disease; (3) current chronic medical illness; (4) a history of head trauma; (5) cardiovascular, pulmonary or systemic disease; (6) claustrophobia experienced during the MRI simulator session; and (7) subjects who could not pitch-match their tinnitus. Twenty-six age-, sex-, education-, and handednessmatched $\mathrm{HC}$ subjects were enrolled as controls. None of the HCs suffered from tinnitus in the past year. Other exclusion criteria were the same as previously described. The characteristics of the subjects are presented in Table 1.

\section{Sound therapy and clinical evaluation}

All of the enrolled tinnitus patients were examined for tinnitus loudness matching $(L=$ loudness of tinnitus), pitch matching ( $T f=$ tinnitus frequency), minimum masking level, and residual inhibition to characterize the tinnitus and prepare for treatment. Narrow band sound therapy was administered to participants in the tinnitus group for 24 weeks, three times a day for 20 minutes each time. For each tinnitus patient, the loudness of sound for treatment was set as $L+5 \mathrm{~dB}$. The frequency was set as a 1 $\mathrm{kHz}$ narrow band when setting $\mathrm{Tf}$ as the middle point of the delivered sound ( $\mathrm{Tf} \pm 0.5 \mathrm{kHz}$; for example, Tf $=4 \mathrm{kHz}$, low sound cut $=3.5 \mathrm{kHz}$, high sound cut $=4.5 \mathrm{kHz}$ ). 
We also asked the patients to fill out the Tinnitus Handicap Inventory (THI) to assess the severity of tinnitus before and after treatment. The primary outcome of this prospective study was the change in THI score after treatment. A reduction of at least 16 points in the THI score was considered effective treatment (41). ${ }^{27}$ The $\mathrm{HC}$ group was not given any kind of sound during the study.

\section{Data acquisition and data preprocessing}

For each patient, to evaluate the change in brain activity under treatment, structural MRI data were collected at baseline and at the end of therapy ( $24^{\text {th }}$ week). The HC group was also scanned at baseline as well as at the $24^{\text {th }}$ week. Images were obtained using a 3.0T MRI system (Prisma, Siemens, Erlangen, Germany) with a 64-channel phase-array head coil. All imaging studies were performed at the Medical Imaging Research Center of our hospital. Parallel imaging was employed for data acquisition. Highresolution three-dimensional (3-D) structural T1-weighted images were acquired using a 3-D magnetization-prepared rapid gradient echo (MP-RAGE) sequence with the following parameters: repetition time $(T R)=2530 \mathrm{~ms}$, echo time $(T E)=2.98 \mathrm{~ms}$, inversion time $(\mathrm{TI})=1100 \mathrm{~ms}, \mathrm{FA}=7^{\circ}$, number of slices $=192$, slice thickness $=1 \mathrm{~mm}$, bandwidth $=240 \mathrm{~Hz} / \mathrm{Px}$, field of view $(\mathrm{FOV})=256 \mathrm{~mm} \times 256 \mathrm{~mm}$, and matrix $=256 \times 256$, resulting in an isotropic voxel size of $1 \mathrm{~mm} \times 1 \mathrm{~mm} \times 1 \mathrm{~mm}^{3}$.

Image preprocessing was performed with the VBM8 toolbox in the SPM8 software package (Statistical Parametric Mapping, Wellcome Department of Cognitive Neurology, London, UK) running in MATLAB (MathWorks, Natick, MA, USA). The procedures for image preprocessing have been described in detail in our previous research (28). Briefly, image processing in this work included spatial normalization using the Montreal Neurological Institute (MNI) 152 template and segmentation of the GM, WM, and cerebrospinal fluid (CSF). Only the GM images were analyzed in this study. Modulation was also applied to avoid volumetric deformation of the GM due to stretching and shrinking effects during the normalization procedure. The modulated GM images were smoothed with a $6 \mathrm{~mm}$ full width at half maximum (FWHM) isotropic Gaussian kernel. Finally, the smoothed GM images were resampled to a $3 \mathrm{~mm} \times 3 \mathrm{~mm} \times 3 \mathrm{~mm}$ voxel size for statistical analysis. Subjects with excessive head motion (more than $1.5 \mathrm{~mm}$ in translation or $1.5^{\circ}$ in rotation) were excluded from analysis.

\section{Construction of SCNs}

First, VBM8 software was used for structural image segmentation. We put GM volume maps of patients before and after treatment in one folder and maps from HCs in another folder. The GM volume value was extracted using DIPABI software (http://rfmri.org/dpabi). Second, the automated anatomical labeling (AAL) atlas was used to divide the whole brain into 90 cortical and subcortical regions of interest (42), and each was considered a network node. Last, we used the Brain Connectivity Toolbox software (43) to construct SCNs in MATLAB version R2017a.

\section{SCN analysis}


We used binarized graphs to calculate global properties and local properties and calculate the area under the curve (AUC) for each property over the sparsity range. The global property was defined as the average inverse of the characteristic path length (44). The global properties included the clustering coefficient, global efficiency, small-world properties, and shortest path length. Local (nodal) structural alterations were evaluated based on the local efficiency, degree centrality and betweenness centrality of each region (45). Nodal efficiency measured the global efficiency of parallel information transfer in a network. Degree centrality is the number of nodes directly connected to the node, which measures the importance of a single node in the network. Betweenness centrality examines the contribution of each node to the shortest path between all other pairs of points (46). The degree centrality and betweenness centrality of nodes reflect the importance of nodes in information transfer.

\section{Statistical analysis}

Demographic data were compared through two-sample $t$ tests and paired two-sample $t$ tests using SPSS 19.0 software (SPSS, Inc., Chicago, IL). $P$ values $<0.05$ were considered statistically significant. Longitudinal changes in the THI score were also analyzed by using paired two-sample $t$ tests.

For VBM data, to determine the group $\times$ time interaction effect between the two groups and the two scans, the main effects of group (the tinnitus patient group and the HC group) and time (baseline and 24week follow-up period), two-way mixed model analysis of variance (ANOVA) and post hoc analyses were performed. An $F$ value of VBM analysis and a $P$ value of $\mathrm{SCN}$ analysis less than 0.05 were considered statistically significant (false discovery rate (FDR) corrected). In post hoc analyses, two-sample $t$ tests and paired two-sample $t$ tests were used to compare GM volumetric changes. To prove our hypothesis, Pearson's correlation analyses were further conducted to investigate the relationship between the change in $\mathrm{GM}$ volume and the clinical characteristics of tinnitus patients (disease duration at baseline, $\triangle \mathrm{THI}$ score $(\triangle T H I$ score $=$ THlpre - THIpost $)) . P<0.05$ was set as the threshold to determine significance. The GM volume results were visualized with BrainNet Viewer (http://www.nitrc.org/projects/bnv/) (47). Pearson's correlation analysis was performed using SPSS 19 software (SPSS, Inc., Chicago, IL) between the THI scores.

For SCNs, we used two-sample $t$ tests to construct a network of patients with tinnitus (baseline and after 24-week treatment) and a healthy group (baseline and after 24-week scan). Then, the graph theory index of the covariant brain network was calculated, and a permutation test (1000 times) was performed on the graph theory index of the two groups. We applied an FDR of $5 \%$ to adjust for the multiple comparisons of mean local and global efficiency across the between-group contrasts before and after 24 weeks of treatment.

\section{Results}

\section{Demographics and behaviors of study participants}


In this study, we enrolled 33 patients with idiopathic tinnitus, and we applied VBM to analyze the GM volume and network changes in the brain in this group before and after sound therapy. Concurrently, 26 $\mathrm{HC}$ individuals were enrolled. Each group of subjects was age-, sex-, and handedness-matched. (Table 1). THI scores were acquired before and after sound therapy. In the data preprocessing step, none of the subjects were excluded according to the head motion criteria. Significant longitudinal decreases in THI scores were observed. The results are summarized in Table 1.

\section{Statistical analysis results}

Brain structural changes between the patient group and $\mathrm{HC}$ group at baseline and either aftertreatment or 24 weeks, respectively, and between the patients before and aftertreatment

As shown in Figure 1 and Table 2, statistical analysis results demonstrated significant differences in GM volume among the tinnitus patients before sound therapy (baseline), tinnitus patients after sound therapy (24 weeks), $\mathrm{HC}$ individuals at baseline and $\mathrm{HC}$ individuals after 24 weeks. These brain regions included the right parahippocampus gyrus, right caudate, left superior temporal gyrus, left cuneus gyrus and right calcarine gyrus (without multiple correction).

Compared to participants in the HC baseline group and HC 24-week group, significantly decreased GM volume was found in the right parahippocampus gyrus, right caudate, left superior temporal gyrus, left cuneus gyrus and right calcarine gyrus of the participants in the tinnitus baseline group (Table 2 and Figure 2).

Compared with the tinnitus baseline group, the 24-week sound therapy tinnitus group demonstrated a significantly higher GM volume in all of the regions mentioned above. Compared with the GM in the HC baseline group, the GM in the HC 24-week group did not reach statistical significance in these brain regions. (Table 2 and Figure 2)

Compared with the $\mathrm{HC}$ baseline group and the $\mathrm{HC}$ 24-week group, the tinnitus sound therapy group demonstrated slightly lower GM volume in the right calcarine gyrus and left cuneus gyrus and slightly higher volume in the right caudate and left superior temporal gyrus; however, these differences did not reach statistical significance (Table 2 and Figure 2).

\section{SCN changes between the patient andHC groups at baseline and either after treatment or after 24 weeks, respectively, and between patients before and aftertreatment}

We calculated the AUC for each network metric, and the AUC provided a summarized scalar for the topological characterization of brain networks. The results showed that there was statistical significance in twelve brain regions (Table 3 and Figure 3), including nonauditory-related and auditory-related brain regions, such as the bilateral rolandic operculum and left superior temporal gyrus, and all twelve regions combined. The left superior temporal gyrus and left rolandic operculum were significantly different in nodal efficiency, nodal degree centrality and nodal betweenness centrality after FDR correction (Table 3 
and Figure 4). Combining the above results, we found that only the left superior temporal gyrus showed significant differences in GM volume and SCNs.

\section{Correlation}

The decreased THI score and GM volume change between these five brain regions were not correlated.

\section{Discussion}

This is a meaningful longitudinal investigation that specifically analyzed the changes in GM volume and SCNs in tinnitus patients at baseline and after 24 weeks of sound therapy. In this study, we found that tinnitus patients had structural changes in the brain after treatment. Anatomical changes in the brain were found in patients before and after sound therapy, mainly in the right parahippocampus gyrus, right caudate, left superior temporal gyrus, left cuneus gyrus and right calcarine gyrus. The results of the SCNs provided crucial information for understanding the network interactions between the whole brain and therapy in tinnitus. To a certain extent, these brain regions can be used as neurobiological targets for tinnitus treatment.

\section{Nonauditory-related structural brain alterations and network performance between patientsand HCs and between patients before and after sound therapy}

We observed a significant increase in GM volume in the right parahippocampus gyrus, right caudate, left cuneus gyrus and right calcarine gyrus in the patients after treatment compared with baseline. The parahippocampal gyrus is regarded to be critical to emotional processing and auditory information storage in tinnitus patients; therefore, some studies have noticed that the parahippocampal gyrus is related to perception in tinnitus patients $(48,49)$. A previous GM study found two major group differences that decreased cortical thickness in the left parahippocampal gyrus in patients with severe tinnitus (50). Our results showed that compared with the tinnitus baseline group, the 24-week sound therapy group demonstrated a significantly higher GM volume in the parahippocampal gyrus. This result showed that after a long period of treatment, the hearing information storage and emotion of tinnitus patients had a certain degree of recovery. The calcarine gyrus is an important part of the primary visual cortex and the main relay station for transmitting retinal signals; thus, changes in the calcarine gyrus may result from patients attending to phantom auditory sensations and having the visual areas contemporaneously activated (51). Our previous research has also shown that the local activity and functional connectivity of the primary auditory cortex were enhanced (52).

In the SCN results, we found that there was a significant difference in the rolandic operculum of nodal efficiency, degree centrality and betweenness centrality. A study speculated that overactivity in the rolandic operculum was associated with middle ear proprioception, and changes in this brain region may suggest that tinnitus could arise as a proprioceptive illusion associated with widespread emotional and somatosensory dysfunction (53). Our results further confirm this inference. Although these SCN changes 
may not be as significant as functional changes, the results also reflect the efficacy of sound therapy in local network properties to a certain extent.

\section{Auditory-related brain structural alterations and network performance between patientsand HCs and between patients before and after sound therapy}

The auditory cortex can be divided into the primary auditory cortex, secondary auditory cortex, and auditory association cortex. Our results indicated increased GM volume in the left superior temporal gyrus, which is the auditory network, mainly overlapping with the auditory association cortex. The abnormal parts of the auditory cortex of tinnitus patients, mainly the auditory association cortex, are relatively sensitive compared to other parts $(12,54,55)$. The left superior temporal gyrus in degree centrality reached a significant difference, indicating the importance of a single node (left superior temporal gyrus) in the network.

Regarding the morphological and functional changes associated with tinnitus, most previous studies and our recent research have focused on functional aspects (56). In this study, the 24-week treatment group showed high levels of auditory-related GM volume, but the tinnitus baseline group showed less auditoryrelated GM volume, which suggests that the associations between networks defined as being within the auditory-related network architecture were generally stronger in the 24-week treatment group. Previous studies have mentioned functional issues but performed fewer structural analyses $(39,57)$. There are structural changes in tinnitus patients, and these structural changes will affect structure after treatment (27); therefore, it is necessary to pay more attention to the structural changes associated with tinnitus patients. The results of this study are important supplements to original research. Therefore, whether in VBM or SCN analyses, the superior temporal gyrus can be used as one of the important structural brain areas to measure the effect of tinnitus treatment.

Compared with previous research on tinnitus, in this study, the aforementioned brain regions could represent new neuroanatomical features of patients with tinnitus. In particular, the superior temporal gyrus, whether in VBM or SCN analyses, can be used as an important structural brain area to measure the effect of tinnitus treatment. Accordingly, combined VBM and SCN analyses can provide novel tools to examine complex network properties of the intact and diseased brain. The two modalities are complementary.

A suitable treatment method and the correct treatment time are the keys to achieving curative effects. In our study, we applied narrow band noise sound therapy with a relatively long treatment time and a relatively better treatment effect. A previous study with the Heidelberg model of music therapy analyzed the average treatment time to be approximately two weeks (37) and found GM volume changes in the precuneus, medial superior frontal areas, and auditory cortex. The analysis in our study was performed for six months of treatment with narrow band noise sound therapy. The morphological changes that may be found are different from those of previous studies, which is supported by our results. However, for different results, we should consider different opinions, which would be conducive to a balanced view of our research. 
There are several limitations of this study. First, the current study is an exploratory study with microstructure changes in $\mathrm{GM}$, and it is difficult to obtain a significant result with a small sample size. Therefore, a threshold of $p<0.001$ (uncorrected for multiple comparisons) was applied in the condition group comparisons based on the stringency of the group contrasts used in this study. In contrast to functional changes, it is difficult for the microstructure itself to change significantly within a relatively short period of time. Therefore, we believe that this result can explain the corresponding scientific problem to a certain extent. Further studies should also include a larger sample size to avoid these problems. Second, many studies have used VBM to better understand tinnitus, but the results are inconsistent. Maybe the methodological differences lead to heterogeneous results (58). We should further pay attention to heterogeneity in future studies. Third, the tinnitus patients included in this study did not have significant hearing loss, which represents only a portion of patients with different types of tinnitus. Last, SCNs have been evaluated with respect to other clinical diseases, but there are few studies on tinnitus, so we should perform more in-depth research in the future.

\section{Conclusion}

This study analyzed the anatomical changes in tinnitus patients before and after treatment for six months. The effect of sound therapy included alterations in brain volume, especially in the left superior temporal lobe. Combined VBM and SCNs can potentially provide us with a better understanding of the neuroanatomical and pathophysiological mechanisms before and after sound therapy in tinnitus patients. Sound therapy had a normalizing effect on tinnitus patients.

\section{Abbreviations}

SCN: structural covariance network

VBM: voxel-based morphometry

HC: healthy control

THI: Tinnitus Handicap Inventory

GM: gray matter

IRB: Institutional Review Board

MP-RAGE: magnetization-prepared rapid gradient echo

TR: repetition time

TE: echo time

TI: inversion time 
FOV: field of view

MNI: Montreal Neurological Institute

CSF: cerebrospinal fluid

AUC: area under the curve

ACC: anterior cingulate cortex

ALFF: amplitude of low-frequency fluctuation

BOLD: blood-oxygen-level dependent

CSF: cerebrospinal fluid signal

rs-fMRI: resting-state functional magnetic resonance imaging

ROI: regions of interest

SPM: Statistical Parametric Mapping

\section{Declarations}

\section{Ethics approval and consent to participate}

This research involved human participants. All authors have declared that this research was approved by the Institutional Review Board (IRB) of Beijing Friendship Hospital, Capital Medical University, Beijing, China. Written informed consent was obtained from all subjects enrolled in this study.

\section{Consent for publication}

Not applicable.

\section{Availability of supporting data}

We agree that the materials described in the manuscript, will be freely available to any scientist wishing to use them for non-commercial purposes, without breaching participant confidentiality.

\section{Competing interests}

The authors declare that there are no conflicts of interests regarding the publication of this paper.

\section{Funding}

Funding information This work was supported by Grant No. 61801311 and No. 61931013 from the National Natural Science Foundation of China, No. [2015] 160 from Beijing Scholars Program, Grant No. 
7182044 from Beijing Natural Science Foundation, No. PX2018001 from Beijing Hospitals Authority, QML20180103 from Beijing Hospitals Authority Youth Programme, No. 2019M660717, No.

2020T130083ZX, No. 2020T130084ZX from China Postdoctoral Science Foundation, No. 2020-Z2-023 from Beijing Postdoctoral Research Foundation, No. yyqdkt2019-31 from Research Foundation of Beijing Friendship Hospital, Capital Medical University.

\section{Author contributions}

XW designed the experiments, performed the statistical analysis, and wrote the manuscript. QC conducted the statistical analysis. PfZ contributed to the manuscript revision. HL, ZW, and CL collected the data. SG, ZY, and ZcW are guarantors of this work. $\mathrm{HL}$ and $\mathrm{ZcW}$ are the corresponding authors of this manuscript. They have full access to all the data in the study and take responsibility for the integrity of the data and the accuracy of the data analysis. All authors contributed to the article and approved the submitted version.

\section{Acknowledgments}

We especially thank Beijing Friendship Hospital, Capital Medical University, for their support with our research. We thank Dr. Shen Liu, from the Department of Otolaryngology Head and Neck Surgery, Beijing Friendship Hospital, Capital Medical University, for their assistance with the data collection and audiological tests.

\section{Authors' information}

${ }^{1}$ Department of Radiology, Beijing Friendship Hospital, Capital Medical University, No.95, Yong An Road, Xicheng District, Beijing 100050, China;

2 Department of Otolaryngology Head and Neck Surgery, Beijing Friendship Hospital, Capital Medical University, Beijing 100050, China

\section{References}

1. Baguley D, McFerran D, Hall D. Tinnitus. Lancet 2013;382:1600-7.

2. Bauer CA. Tinnitus. N Engl J Med 2018;378:1224-31.

3. Shore SE, Roberts LE, Langguth B. Maladaptive plasticity in tinnitus-triggers, mechanisms and treatment. Nat Rev Neurol 2016;12:150-60.

4. Schecklmann M, Pregler M, Kreuzer PM, Poeppl TB, Lehner A, Cronlein T, Wetter TC, Frank E, Landgrebe M, Langguth B. Psychophysiological Associations between Chronic Tinnitus and Sleep: A Cross Validation of Tinnitus and Insomnia Questionnaires. Biomed Res Int 2015;2015:461090.

5. Dobie RA. Depression and tinnitus. Otolaryngol Clin North Am 2003;36:383-8.

6. Heller AJ. Classification and epidemiology of tinnitus. Otolaryngol Clin North Am 2003;36:239-48. 
7. Ryu CW, Park MS, Byun JY, Jahng GH, Park S. White matter integrity associated with clinical symptoms in tinnitus patients: A tract-based spatial statistics study. Eur Radiol 2016;26:2223-32.

8. Han L, Na Z, Chunli L, Yuchen C, Pengfei Z, Hao W, Xu C, Peng Z, Zheng W, Zhenghan Y, Shusheng G, Zhenchang W. Baseline Functional Connectivity Features of Neural Network Nodes Can Predict Improvement After Sound Therapy Through Adjusted Narrow Band Noise in Tinnitus Patients. Front Neurosci 2019;13:614.

9. Schmidt SA, Carpenter-Thompson J, Husain FT. Connectivity of precuneus to the default mode and dorsal attention networks: A possible invariant marker of long-term tinnitus. Neuroimage Clin 2017;16:196-204.

10. Chen Q, Wang Z, Lv H, Zhao P, Yang Z, Gong S, Wang Z. Reorganization of Brain White Matter in Persistent Idiopathic Tinnitus Patients Without Hearing Loss: Evidence From Baseline Data. Front Neurosci 2020;14:591.

11. Han L, Zhaohui L, Fei Y, Pengfei Z, Ting L, Cheng D, Zhenchang W. Disrupted neural activity in unilateral vascular pulsatile tinnitus patients in the early stage of disease: evidence from restingstate fMRI. Prog Neuropsychopharmacol Biol Psychiatry 2015;59:91-9.

12. Lv H, Zhao P, Liu Z, Li R, Zhang L, Wang P, Yan F, Liu L, Wang G, Zeng R, Li T, Dong C, Gong S, Wang $Z$. Abnormal resting-state functional connectivity study in unilateral pulsatile tinnitus patients with single etiology: A seed-based functional connectivity study. Eur J Radiol 2016;85:2023-9.

13. Lv H, Zhao P, Liu Z, Wang G, Zeng R, Yan F, Dong C, Zhang L, Li R, Wang P, Li T, Gong S, Wang Z. Frequency-Dependent Neural Activity in Patients with Unilateral Vascular Pulsatile Tinnitus. Neural Plast 2016;2016:4918186.

14. Lv H, Zhao P, Liu Z, Liu X, Ding H, Liu L, Wang G, Xie J, Zeng R, Chen Y, Yang Z, Gong S, Wang Z. Lateralization effects on functional connectivity of the auditory network in patients with unilateral pulsatile tinnitus as detected by functional MRI. Prog Neuropsychopharmacol Biol Psychiatry 2018;81:228-35.

15. Hofmeier B, Wolpert S, Aldamer ES, Walter M, Thiericke J, Braun C, Zelle D, Ruttiger L, Klose U, Knipper M. Reduced sound-evoked and resting-state BOLD fMRI connectivity in tinnitus. Neuroimage Clin 2018;20:637-49.

16. van der Loo E, Congedo M, Vanneste S, Van De Heyning P, De Ridder D. Insular lateralization in tinnitus distress. Auton Neurosci 2011;165:191-4.

17. Vanneste S, De Ridder D. The auditory and non-auditory brain areas involved in tinnitus. An emergent property of multiple parallel overlapping subnetworks. Front Syst Neurosci 2012;6:31.

18. Vanneste SS, Heyning PVD, Ridder DD. Contralateral parahippocampal gamma-band activity determines noise-like tinnitus laterality: A region of interest analysis. Neuroscience 2011;199:481-90.

19. Vanneste S, Plazier M, der Loo E, de Heyning PV, Congedo M, De Ridder D. The neural correlates of tinnitus-related distress. Neuroimage 2010;52:470-80.

20. Dirk DR, Sven V, Marco C, Thomas K. The Distressed Brain: A Group Blind Source Separation Analysis on Tinnitus. Plos One 2011;6:e24273. 
21. Besteher B, Gaser C, Ivansic D, Guntinas-Lichius O, Dobel C, Nenadic I. Chronic tinnitus and the limbic system: Reappraising brain structural effects of distress and affective symptoms. Neuroimage Clin 2019;24:101976.

22. Tae WS, Yakunina N, Lee WH, Ryu YJ, Ham HK, Pyun SB, Nam EC. Changes in the regional shape and volume of subcortical nuclei in patients with tinnitus comorbid with mild hearing loss. Neuroradiology 2018;60:1203-11.

23. Nemoto K. [Understanding Voxel-Based Morphometry]. Brain Nerve 2017;69:505-11.

24. Ashburner J, Friston KJ. Voxel-Based Morphometry-The Methods. Neuroimage 2000;11:805-21.

25. Husain FT, Medina RE, Davis CW, Szymko-Bennett Y, Simonyan K, Pajor NM, Horwitz B. Neuroanatomical changes due to hearing loss and chronic tinnitus: a combined VBM and DTI study. Brain Res 2011;1369:74-88.

26. Meyer M, Neff P, Liem F, Kleinjung T, Weidt S, Langguth B, Schecklmann M. Differential tinnitusrelated neuroplastic alterations of cortical thickness and surface area. Hear Res 2016;342:1-12.

27. Cheng S, Xu G, Zhou J, Qu Y, Li Z, He Z, Yin T, Ma P, Sun R, Liang F. A Multimodal Meta-Analysis of Structural and Functional Changes in the Brain of Tinnitus. Front Hum Neurosci 2020;14:28.

28. Liu Y, Lv H, Zhao P, Liu Z, Chen W, Gong S, Wang Z, Zhu JM. Neuroanatomical Alterations in Patients with Early Stage of Unilateral Pulsatile Tinnitus: A Voxel-Based Morphometry Study. Neural Plast 2018;2018:4756471.

29. Richmond S, Beare R, Johnson KA, Allen NB, Seal ML, Whittle S. Structural covariance networks in children and their associations with maternal behaviors. Neuroimage 2019;202:115965.

30. Drenthen GS, Backes WH, Rouhl RPW, Vlooswijk MCG, Majoie M, Hofman PAM, Aldenkamp AP, Jansen JFA. Structural covariance networks relate to the severity of epilepsy with focal-onset seizures. Neuroimage Clin 2018;20:861-7.

31. Poeppl TB, Langguth B, Lehner A, Frodl T, Rupprecht R, Kreuzer PM, Landgrebe M, Schecklmann M. Brain stimulation-induced neuroplasticity underlying therapeutic response in phantom sounds. Hum Brain Mapp 2018;39:554-62.

32. Zenner HP, Delb W, Kroner-Herwig B, Jager B, Peroz I, Hesse G, Mazurek B, Goebel G, Gerloff C, Trollmann R, Biesinger E, Seidler H, Langguth B. A multidisciplinary systematic review of the treatment for chronic idiopathic tinnitus. Eur Arch Otorhinolaryngol 2017;274:2079-91.

33. Langguth B, Kreuzer PM, Kleinjung T, De Ridder D. Tinnitus: causes and clinical management. Lancet Neurol 2013;12:920-30.

34. Yakunina N, Lee WH, Ryu YJ, Nam EC. Tinnitus Suppression Effect of Hearing Aids in Patients With High-frequency Hearing Loss: A Randomized Double-blind Controlled Trial. Otol Neurotol 2019;40:865-71.

35. Olze H. [Cochlear implants and tinnitus]. HNO 2015;63:291-7.

36. Lee SY, Rhee J, Shim YJ, Kim Y, Koo JW, De Ridder D, Vanneste S, Song JJ. Changes in the RestingState Cortical Oscillatory Activity 6 Months After Modified Tinnitus Retraining Therapy. Front 
Neurosci 2019;13:1123.

37. Krick CM, Grapp M, Daneshvar-Talebi J, Reith W, Plinkert PK, Bolay HV. Cortical reorganization in recent-onset tinnitus patients by the Heidelberg Model of Music Therapy. Front Neurosci 2015;9:49.

38. Henry JA, Schechter MA, Nagler SM, Fausti SA. Comparison of tinnitus masking and tinnitus retraining therapy. J Am Acad Audiol 2002;13:559-81.

39. Han L, Yawen L, Hao W, Chunli L, Pengfei Z, Zhengyu Z, Zhaodi W, Zhenghan Y, Shusheng G, Zhenchang W. Effects of sound therapy on resting-state functional brain networks in patients with tinnitus: A graph-theoretical-based study. J Magn Reson Imaging 2019;50:1731-41.

40. Krick CM, Argstatter H, Grapp M, Plinkert PK, Reith W. Heidelberg Neuro-Music Therapy Enhances Task-Negative Activity in Tinnitus Patients. Front Neurosci 2017;11:384.

41. Zeman F, Koller M, Figueiredo R, Aazevedo A, Rates M, Coelho C, Kleinjung T, de Ridder D, Langguth B, Landgrebe $\mathrm{M}$. Tinnitus handicap inventory for evaluating treatment effects: which changes are clinically relevant? Otolaryngol Head Neck Surg 2011;145:282-7.

42. Tzourio-Mazoyer N, Landeau B, Papathanassiou D, Crivello F, Etard O, Delcroix N, Mazoyer B, Joliot M. Automated anatomical labeling of activations in SPM using a macroscopic anatomical parcellation of the MNI MRI single-subject brain. Neuroimage 2002;15:273-89.

43. Rubinov M, Sporns O. Complex network measures of brain connectivity: uses and interpretations. Neuroimage 2010;52:1059-69.

44. Bullmore E, Sporns O. Complex brain networks: graph theoretical analysis of structural and functional systems. Nat Rev Neurosci 2009;10:186-98.

45. Fortanier E, Grapperon AM, Le Troter A, Verschueren A, Ridley B, Guye M, Attarian S, Ranjeva JP, Zaaraoui W. Structural Connectivity Alterations in Amyotrophic Lateral Sclerosis: A Graph Theory Based Imaging Study. Front Neurosci 2019;13:1044.

46. Ravasz E, Barabasi AL. Hierarchical organization in complex networks. Phys Rev E Stat Nonlin Soft Matter Phys 2003;67:026112.

47. Xia M, Wang J, He Y. BrainNet Viewer: a network visualization tool for human brain connectomics. PLoS One 2013;8:e68910.

48. Leaver AM, Turesky TK, Seydell-Greenwald A, Morgan S, Kim HJ, Rauschecker JP. Intrinsic network activity in tinnitus investigated using functional MRI. Hum Brain Mapp 2016;37:2717-35.

49. Vanneste S, De Ridder D. Deafferentation-based pathophysiological differences in phantom sound: Tinnitus with and without hearing loss. Neuroimage 2016;129:80-94.

50. Schmidt SA, Zimmerman B, Bido Medina RO, Carpenter-Thompson JR, Husain FT. Changes in gray and white matter in subgroups within the tinnitus population. Brain Res 2018;1679:64-74.

51. Zhou GP, Shi XY, Wei HL, Qu LJ, Yu YS, Zhou QQ, Yin X, Zhang H, Tao YJ. Disrupted Intraregional Brain Activity and Functional Connectivity in Unilateral Acute Tinnitus Patients With Hearing Loss. Front Neurosci 2019;13:1010. 
52. Lv H, Zhao P, Liu C, Wang Z, Wang X, Chen Q, Gong S, Wang Z. The effects of sound therapy in tinnitus are characterized by altered limbic and auditory networks. Brain Communications 2020;In press.

53. Job A, Pons Y, Lamalle L, Jaillard A, Buck K, Segebarth C, Delon-Martin C. Abnormal cortical sensorimotor activity during "Target" sound detection in subjects with acute acoustic trauma sequelae: an fMRI study. Brain Behav 2012;2:187-99.

54. Zhang J, Chen YC, Feng X, Yang M, Liu B, Qian C, Wang J, Salvi R, Teng GJ. Impairments of thalamic resting-state functional connectivity in patients with chronic tinnitus. Eur J Radiol 2015;84:1277-84.

55. Chen YC, Zhang J, Li XW, Xia W, Feng X, Gao B, Ju SH, Wang J, Salvi R, Teng GJ. Aberrant spontaneous brain activity in chronic tinnitus patients revealed by resting-state functional MRI. Neuroimage Clin 2014;6:222-8.

56. Minami SB, Oishi N, Watabe T, Uno K, Ogawa K. Auditory Related Resting State fMRI Functional Connectivity in Tinnitus Patients: Tinnitus Diagnosis Performance. Otol Neurotol 2018;39:1-5.

57. Berlot E, Arts R, Smit J, George E, Gulban OF, Moerel M, Stokroos R, Formisano E, De Martino F. A 7 Tesla fMRI investigation of human tinnitus percept in cortical and subcortical auditory areas. Neuroimage Clin 2020;25:102166.

58. Scott-Wittenborn N, Karadaghy OA, Piccirillo JF, Peelle JE. A methodological assessment of studies that use voxel-based morphometry to study neural changes in tinnitus patients. Hear Res 2017;355:23-32.

\section{Tables}

TABLE 1. Demographic and Clinical Characteristics of Participants 


\begin{tabular}{|c|c|c|c|c|c|}
\hline Characteristics & $\begin{array}{l}\text { Tinnitus } \\
\text { patients } \\
\text { (baseline, } n= \\
\text { 33) }\end{array}$ & $\begin{array}{l}\text { Tinnitus } \\
\text { patients } \\
\left(24^{\text {th }} \text { week, }\right. \\
n=33)\end{array}$ & $\begin{array}{l}\text { Healthy } \\
\text { controls } \\
\text { (baseline, } \mathrm{n}= \\
26 \text { ) }\end{array}$ & $\begin{array}{l}\text { Healthy } \\
\text { controls } \\
\left(24^{\text {th }} \text { week, }\right. \\
\mathrm{n}=26)\end{array}$ & $\begin{array}{l}P \\
\text { value }\end{array}$ \\
\hline Age (years, $\pm s$ ) & $48.2 \pm 12.4$ & & $47.3 \pm 9.6$ & & $0.745^{\mathrm{a}}$ \\
\hline $\begin{array}{l}\text { Gender } \\
\text { (male/female) }\end{array}$ & $23 / 10$ & & $15 / 11$ & & $>0.99^{b}$ \\
\hline Handedness & $\begin{array}{l}33 \text { right- } \\
\text { handed }\end{array}$ & & $\begin{array}{l}26 \text { right- } \\
\text { handed }\end{array}$ & & $>0.99^{a}$ \\
\hline $\begin{array}{l}\text { Tinnitus duration } \\
\text { (months) }\end{array}$ & $\geq 6 \& \leq 48$ & & NA & & NA \\
\hline Tinnitus pitch & $\begin{array}{l}250 \sim 8,000 \\
\mathrm{~Hz}\end{array}$ & & & & NA \\
\hline THI score & $52.5 \pm 44.3$ & $37.3 \pm 20.9$ & NA & NA & $0.011^{c}$ \\
\hline$\triangle T H I$ score & $15.3 \pm 32.8$ & NA & NA & NA & NA \\
\hline Normal hearing & All & & All & & NA \\
\hline
\end{tabular}

Data are presented as mean \pm standard deviation for all variables except gender

THI: Tinnitus Handicap Inventory.

$\triangle T H I=T H I p r e-T H I p o s t . ~ N A:$ not applicable

a Two-sample $t$ tests

b Chi-square test

c Paired-samples $t$-tests

TABLE 2.Regions Showed a Significant Difference in Volumes between Idiopathic Tinnitus Patients Before and After 24 Weeks of Sound Therapy and the Controls

\begin{tabular}{|c|c|c|c|c|c|c|}
\hline \multirow[t]{2}{*}{ Brain regions } & & \multicolumn{3}{|c|}{ Peak location } & \multirow[t]{2}{*}{ Peak F-score } & \multirow[t]{2}{*}{ Voxel numbers } \\
\hline & & $x$ & $y$ & z & & \\
\hline Parahippocampus gyrus & $\mathrm{R}$ & 25 & -18 & -25 & 29.62 & 541 \\
\hline Caudate & $\mathrm{R}$ & 16 & 7 & 14 & 20.04 & 438 \\
\hline Superior temporal gyrus & L & -47 & -23 & 8 & 15.15 & 117 \\
\hline Cuneus gyrus & $\mathrm{L}$ & 0 & -77 & 22 & 15.96 & 371 \\
\hline Calcarine gyrus & $\mathrm{R}$ & 14 & -88 & 6 & 20.57 & 441 \\
\hline
\end{tabular}


TABLE 3.Regions Showed a Significant Difference in network

( $P<0$ 05, FDR corrected, 1000 permutations)

\begin{tabular}{|c|c|c|c|c|}
\hline \multirow[t]{2}{*}{ Characteristics } & AAL & $P$ value & AAL & $P$ value \\
\hline & \multicolumn{2}{|l|}{ Brain Regions } & \multicolumn{2}{|l|}{ Brain Regions } \\
\hline \multirow[t]{6}{*}{ Nodal Efficiency } & 5_Frontal_Sup_Orb_L & 0.001 & 17_Rolandic_Oper_L & $0.000^{a}$ \\
\hline & 18_Rolandic_Oper_R & 0.002 & 44_Calcarine_R & 0.024 \\
\hline & 46_Cuneus_R & 0.029 & 48_Lingual_R & 0.049 \\
\hline & 53_Occipital_Inf_L & 0.01 & 81_Temporal_Sup_L & $0.000^{\mathrm{a}}$ \\
\hline & 83_Temporal_Pole_Sup_L & 0.023 & 84_Temporal_Pole_Sup_R & 0.005 \\
\hline & 89_Temporal_Inf_L & 0.028 & 90_Temporal_Inf_R & 0.043 \\
\hline \multirow[t]{6}{*}{ Nodal DC } & 5_Frontal_Sup_Orb_L & 0.001 & 17_Rolandic_Oper_L & $0.000^{\mathrm{a}}$ \\
\hline & 18_Rolandic_Oper_R & 0.002 & 44_Calcarine_R & 0.024 \\
\hline & 46_Cuneus_R & 0.029 & 48_Lingual_R & 0.049 \\
\hline & 53_Occipital_Inf_L & 0.023 & 81_Temporal_Sup_L & $0.000^{a}$ \\
\hline & 83_Temporal_Pole_Sup_L & 0.023 & 84_Temporal_Pole_Sup_R & 0.005 \\
\hline & 89_Temporal_Inf_L & 0.028 & 90_Temporal_Inf_R & 0.043 \\
\hline \multirow[t]{6}{*}{ Nodal BC } & 5_Frontal_Sup_Orb_L & 0.001 & 17_Rolandic_Oper_L & $0.000^{a}$ \\
\hline & 18_Rolandic_Oper_R & 0.002 & 44_Calcarine_R & 0.024 \\
\hline & 46_Cuneus_R & 0.029 & 48_Lingual_R & 0.049 \\
\hline & 53_Occipital_Inf_L & 0.01 & 81_Temporal_Sup_L & $0.000^{\mathrm{a}}$ \\
\hline & 83_Temporal_Pole_Sup_L & 0.023 & 84_Temporal_Pole_Sup_R & 0.005 \\
\hline & 89_Temporal_Inf_L & 0.028 & 90_Temporal_Inf_R & 0.043 \\
\hline
\end{tabular}

a: FDR corrected; DC: Degree Centrality; BC: Betweenness Centrality

\section{Figures}




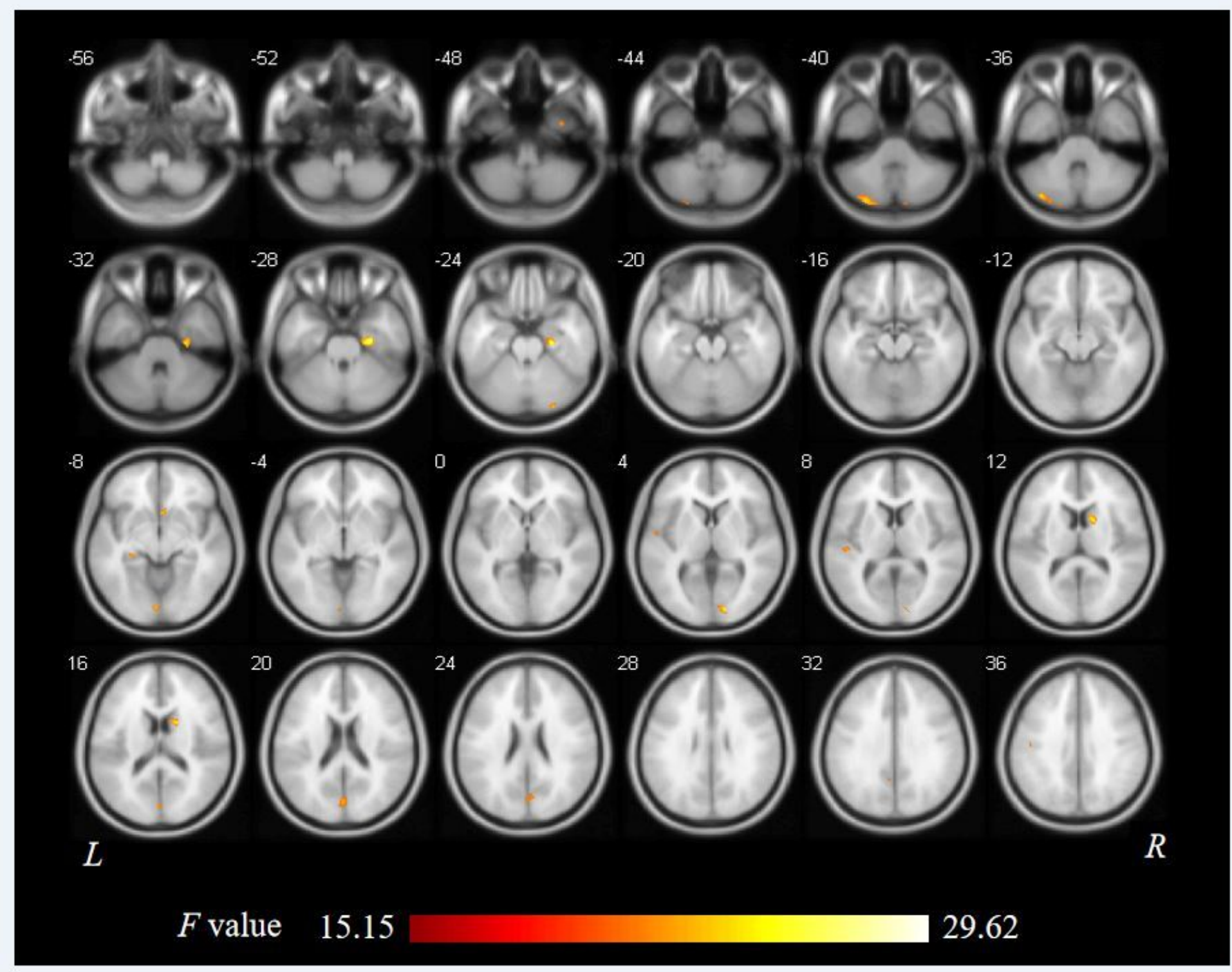

\section{Figure 1}

ANOVA differences in gray matter volume (GMV) changed among patients at baseline, patients after 24 weeks of sound treatment, HCs at baseline, and HCs after 24 weeks ( $P<0.05$; L left, R right). The results showed differences in GM volume in the right parahippocampus gyrus, right caudate, left superior temporal gyrus, left cuneus gyrus and right calcarine gyrus, which are shown in red. L, left; R, right; 
Right Calcarine Gyrus

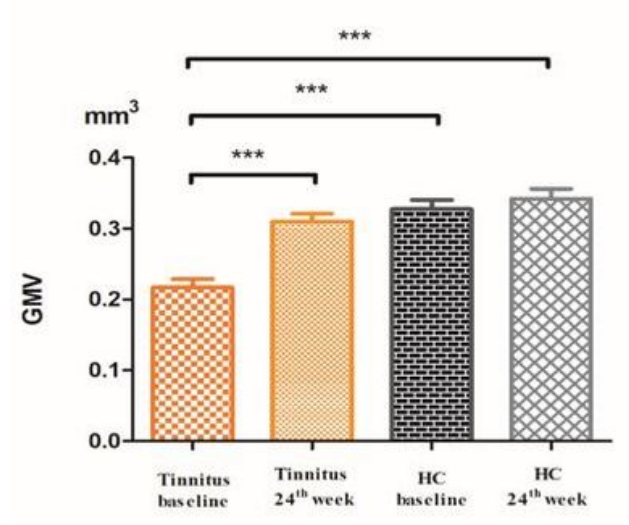

Right Caudate

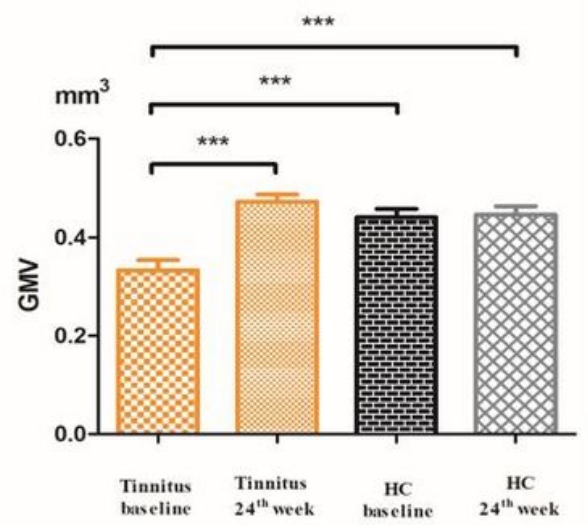

Left Cuneus Gyrus

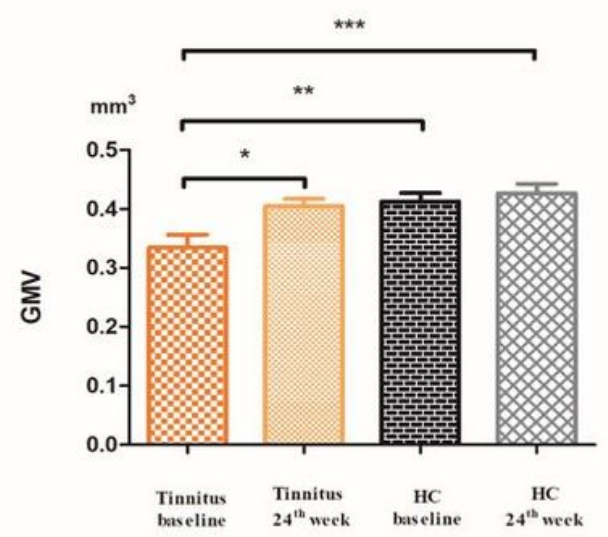

Right Parahippocampus Gyrus

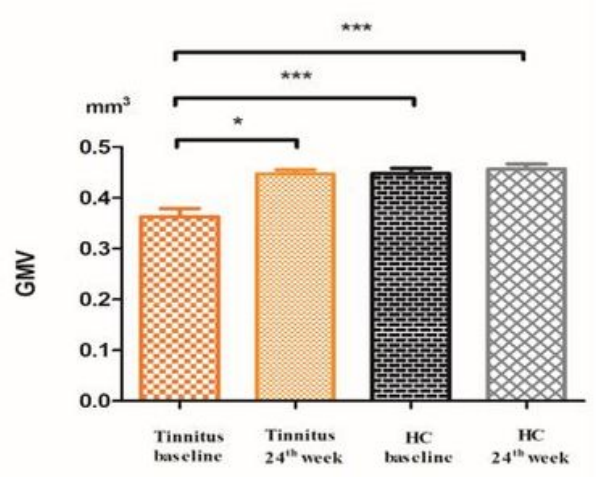

Left Superior Temporal Gyrus

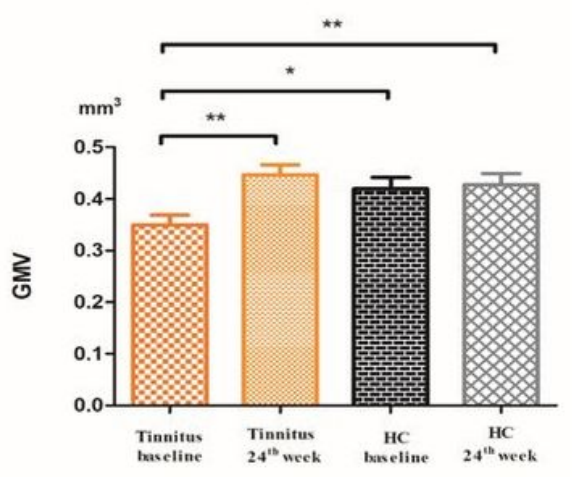

Figure 2

The post hoc analysis showed that the GMV changed among the baseline, 24-week sound treatment, $\mathrm{HC}$ baseline, and HC 24-week groups. ${ }^{*} \mathrm{P}<0.05,{ }^{*} \mathrm{P}<0.01$, ${ }^{* \star} \mathrm{P}<0.001$. The $\mathrm{P}$ values are not corrected for multiple comparisons. 

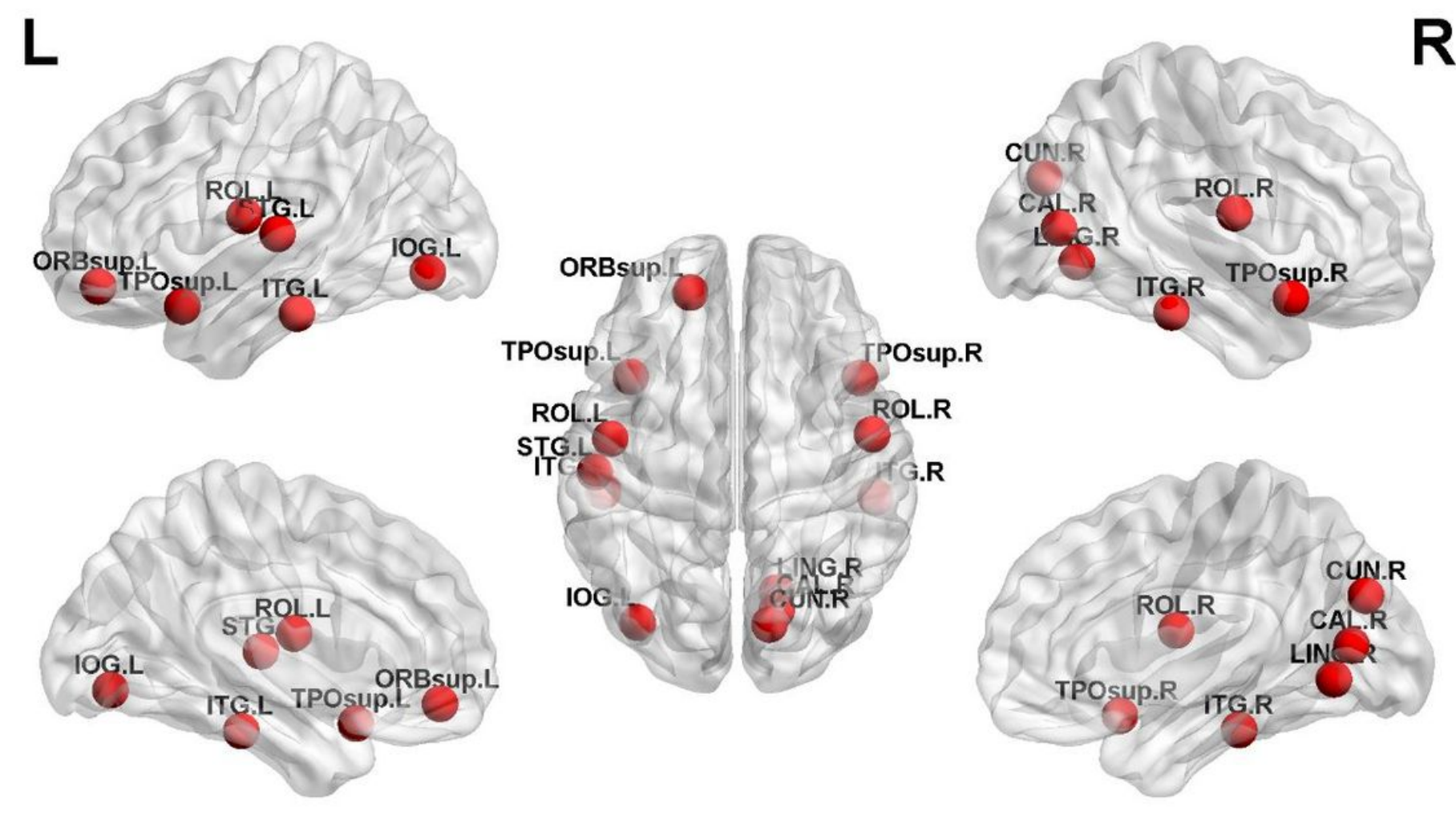

Figure 3

Twelve brain regions reached statistical significance in nodal efficiency and nodal betweenness centrality of the AAL-90 SCN in patients before and after treatment, in HCs at baseline and in HCs after 24 weeks (red ball, $\mathrm{P} \otimes 0.05$ ). The results were produced using permutation testing and visualized using the BrainNet Viewer (NKLCNL, Beijing Normal University). Three-dimensional representations (left: lateral and medial view of the left hemisphere; center: dorsal view; right: lateral and medial view of the right hemisphere) show between-group differences in nodal efficiency, degree centrality and nodal betweenness centrality, according to their centroid stereotaxic coordinates. A list of anatomical labels for the nodes is in Table 3. 

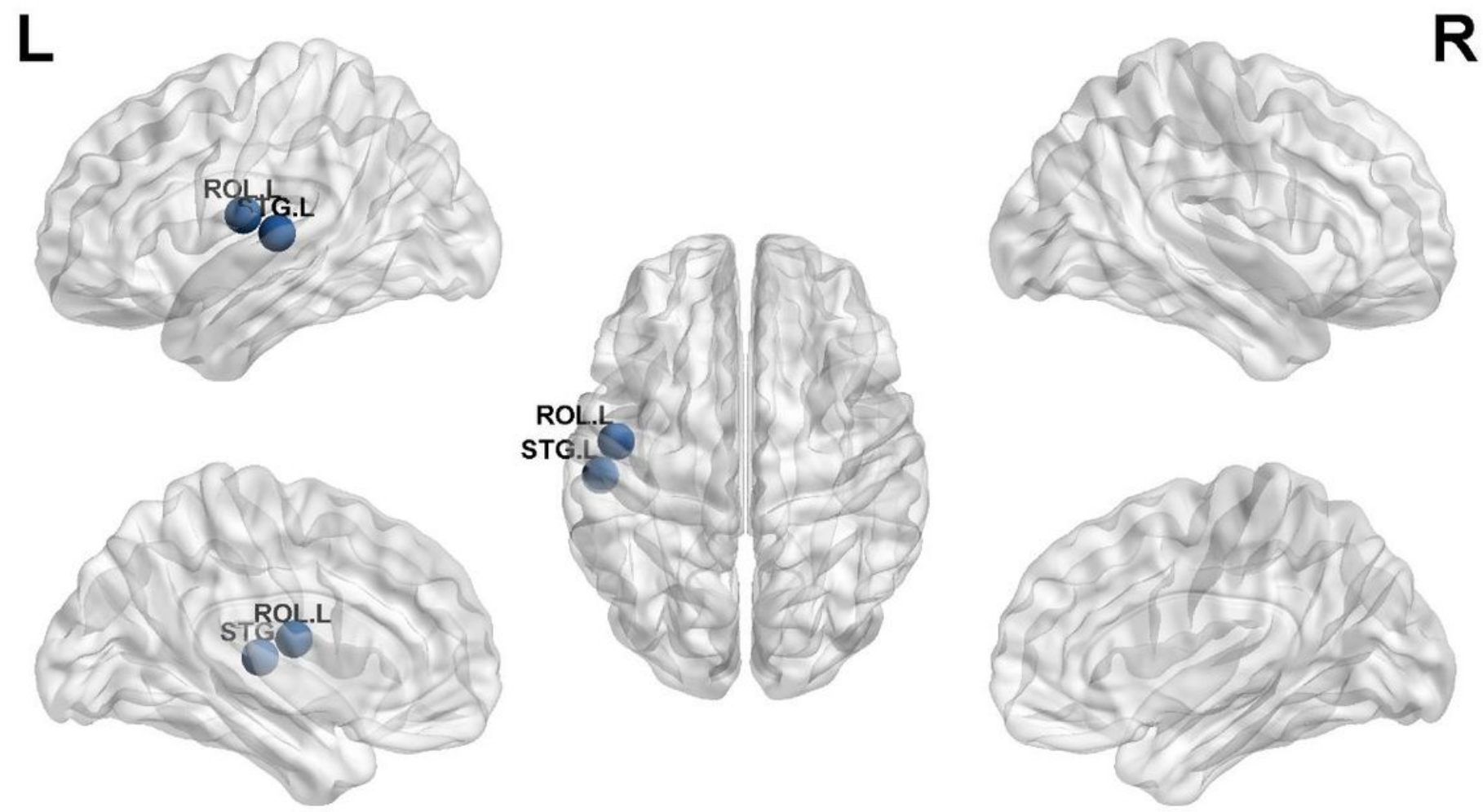

\section{Figure 4}

The left superior temporal gyrus and left rolandic operculum were significantly different in nodal efficiency, nodal degree centrality and nodal betweenness centrality after FDR correction (blue ball, $\mathrm{P} \rrbracket$ $0.05)$. 